in less than a minute, and which makes a minimal opening; we believe that a trephine opening is needless even if it is desired to introduce a considerable quantity of fluid. For the present, at all events, we prefer serum salvarsanised in vitro by adding 40 or 50 milligrammes of neosalvarsan to 5 c.c. of serum obtained from the patient and rendered inactive. That is the dosis optima tolerated by the brain and one which very rapidly arrests the movements of the treponema pallidum. ...... The injections should be limited to the sub. arachnoid space and should not damage the cerebral cortex."

It will be seen that Marinesco and Minea refer to Förster as having injected serum into the ventricles of general paralytics, but in the Münchener Medicinische Wochenschrift ${ }^{4}$ a different reading of Förster's paper is given as follows : "Förster read a paper at the Congress at Jena (Nov. 1st and 2nd, 1913) on Some Inoculation Experiments. He obtained by cranial puncture fragments of the brains of general paralytics, and planted them in guinea-pigs and monkeys. These fragments of the cerebral cortex contained active spirochætes in large numbers. He concluded by saying that "we might take advantage of the wellknown susceptibility of the spirochate to the antibodies in the blood by bringing these bodies into direct contact with the brain by injecting the patient's own serum into the subdural space or the ventricle." Unfortunately, I have not yet been able to obtain a copy of the transactions of the Jena Congress. The plan of cranial or orbital puncture for the introduction of remedies into the cranial cavity does in no way commend itself to me. The remedy in these desperate cases should be given in a scientific and precise manner. The "minimal opening" seems to me utterly wrong. It is impossible with a " minimal opening" of the cranial cavity or by passing a hollow needle through the sphenoidal fissure ${ }^{5}$ to know exactly where the remedy has been injected. A trephine opening and a deliberate operative method is the only way at present known of scientifically injecting remedies into the cranial cavity. The circle of bone if thought desirable can be replaced at the conclusion of the operation. Desperate diseases require desperate remedies if any good is to be attained. Some may think apparently that a trephine opening is a desperate measure, but no one with any experience of modern cranial surgery would give his assent to such a view.

Harley-street, w.

* 1913, p. 2700 .

5 Beriel : Neurologisches Centralblatt, 1914.

Post-Graduate College, West London HosPITAL. - A conversazione was held at the West London Hospital on Wednesday evening, May 20th. The guests were received by the senior surgeon (Mr. A. Baldwin) and the dean of the college (Mr. Donald J. Armour). An admirable musical performance was given by the string band of King Edward's Horse, and kinematographic views of the Panama Canal and of various interesting continental localities were shown. Some really excellent drawing-room magic was provided by Mr. Herbert J. Collings. There were exhibitions of optical, surgical, ophthalmic, $\mathrm{X}$ ray, and other scientific instruments, drugs, and medical publications. Dr. R. R. Flworthy showed favus and other preparations, and Dr. Reginald Morton stereoscopic and other radiographs. Refreshments were provided in the lecture-room.

\section{THE TREATMENT OF PARENCHYMATOUS SYPHILIS BY INTRACRANIAL MEDICATION.}

BY HARRY CAMPBELL, M.D. LOND., F.R.C.P.LOND., PHYSICIAN TO THE WEST END HOSPITAL FOR DISEASES

\author{
THE AERVOUS SYSTEM, ETO.
}

SyPHILITIC diseases of the central nervous system fall under two heads-those in which the spirochrtes infest the meninges and the walls of the blood-vessels, producing such lesions as gummata, meningitis, and endarteritis; and those in which the parasites get lodged between the nerve cells (i.e., in the perineuronic $1 \mathrm{ymph}$ spaces), giving rise to a primary parenchymatous degeneration, of which dementia paralytica, tabes dorsalis, and tabo-paresis are the commonest manifestations.

These two varieties of syphilis of the nervous system-the interstitial and the parenchymatous, as we may term them-exhibit many differences. The most noteworthy of these is that which relates to the effect of treatment upon them. While the interstitial variety is in large measure amenable to antisyphilitic remedies (salvarsan, mercury, potassium iodide) conveyed through the blood, parenchymatous syphilis, on the other hand, is wholly, or all but wholly, uninfluenced by treat. ment of this kind. The explanation probably lies in the fact that the drugs in question are incapable of percolating the walls of the capillaries which supply the lymph environing the nerve cells of the central nervous system, and thus are unable to reach the spirochætes ensconced between these cells.

The problem which confronts us in the treatment of parenchymatous syphilis would thus seem to be, how to get our spirillicidal remedies conveyed to the perineuronic lymph spaces. A ready means presents itself. All that is necessary is to intro. duce them into the subarachnoid space, either directly, or indirectly via the cerebral ventricles. Dr. F. W. Mott has long taught that the perineuronic lymph and the cerebro-spinal fluid are practically the same; and Dr. F. L. Golla has recently shown that a certain amount of the cerebro-spinal fluid in the subarachnoid space does, in point of fact, flow by way of the perivascular lymph spaces of the brain and spinal cord into the perineuronic lymph spaces, whence it drains off into the capillary venules. The method he adopts is to inject the subarachnoid cavity with colloidal carbon, allowing the animal to survive the injection some days, so as to give time for the translation of the carbon particles. These do not pass through animal membranes, and thus can only travel along open channels. Dr. Golla has kindly afforded me the opportunity of examining microscopically sections of the brain and spinal cord of animals thus treated, and it has been quite easy to trace the dark particles along the perivascular sheaths into the perineuronic lymph spaces, and even indeed into the nerve cells themselves. We thus see that, though spixillicidal substances cannot be conveyed to the perineuronic spaces through the blood stream, they can be made to reach these spaces by the route described, thus coming to grips with the spirochætes.

A question of importance in connexion with this mode of treatment is the direction in which the cerebro-spinal fluid flows within the subarachnoid space. Does the direction of the flow, e.g., permit 
of our remedies, when introduced into the spinal sac, reaching the cerebral cortex?

The cerebro-spinal fluid appears to be secreted partly by the cells covering the choroid plexuses within the ventricles, and partly by those lining the subarachnoid space. That derived from the ventricles escapes into the subarachnoid space by certain apertures, notably by the foramen of Majendie in the roof of the fourth ventricle-i.e., it passes chiefly, if not entirely, into the lower, subtentorial cranial chamber. As regards the flow of the cerebro-spinal fluid contained in the subarachnoid space, it is probably sluggish, moving mainly caudal-wards, though influenced somewhat by gravity. The flow is probably in large measure determined by the rhythmic pulsations (cardiac and respiratory) of the brain and spinal cord. These by commoting the fluid lead to an ebb and flow at the foramen magnum, the incisura tentorii (connecting the upper and lower cranial chambers), and the various orifices between the ventricles and the subarachnoid space. Hence some interdrainage of the fluid is continually taking place between the spinal sac and the lower cerebral chamber (containing the cerebellum, medulla, and pons), between the lower and the upper cerebral chambers, and again between the ventricles and the cranial subarachnoid space. In short, these rhythmic commotions lead to such a churning up of the fluid that a substance introduced into any part of the subarachnoid space (supra-tentorial, infra-tentorial, or spinal) tends to diffuse itself throughout the entire space. Thus when colloidal carbon is introduced subdurally above the tentorium it blackens the entire brain and spinal cord, and even to some extent the interior of the ventricles. The like happens when it is injected into the spinal arachnodural sac, though in this case little blackening of the cerebral hemispheres occurs. Indeed, Goldmann, after injecting trypan blue into the spinal sac, found them unstained. Golla, on the other hand, employing colloidal carbon and allowing some days to elapse before destroying the animal, found definite blackening of the hemispheres.

It would appear from these injection experiments that a considerable interchange of the fluid takes place between the spinal sac and the lower cranial chamber (at the foramen magnum), but that as regards the upper and the lower cranial chambers (communicating with one another at the incisura tentorii) the flow is mainly caudal-wards; and that while it is possible to convey our medicaments more or less evenly throughout the entire cerebro. spinal axis by introducing them into the upper cranial subarachnoid space, and also to reach the entire spinal cord, bulb, and cerebellum, by introducing them through the spinal sac, it is not possible by this latter method to convey more than a very small fraction of them to the cerebral hemispheres. This explains why in tabes intraspinal injections usually suffice, and why in general paralysis they are comparatively useless. If we rely upon intraspinal injections in the latter disease, the flow of the cerebro-spinal fluid from the spinal sac into the cranial cavity should be encouraged by raising the foot of the bed and allowing the patient's head to be at a lower level than the sacrum; also by urging him to turn about from side to side, and thus by commotion of the fluid to favour the diffusion of its spirillicidal contents. But even so we shall not succeed in carrying more than a very small proportion of the injections to the main seat of the spirochætes-the cerebral cortex. (It must not be forgotten, in considering the beneficial results of intraspinal injections in dementia paralytica, that the lesions in this disease are not confined to the cerebrum, but that they also affect the cerebellum, bulb, and spinal cord.)

It was the consciousness of our inability to convey our remedies in sufficient quantity to the cerebral cortex by the intraspinal route that led me to approach Mr. C. A. Ballance with regard to the possibility of introducing them directly into the cranial cavity. I had seen such striking results from the employment of intraspinal injections of salvarsanised serum in tabes as to make me hopeful: of equally good results in dementia paralytica, if only the serum could be conveyed in sufficient quantity to the cerebral cortex. I had hoped that it could be introduced directly into the subarachnoid space of the upper cranial cavity by means of a small trephine opening, but Mr. Ballance has con. vinced me, by actual demonstration on the operating table, that it is very difficult, if not impossible, to introduce more than a rery small quantity of fuid in this way. For this method to be successful we shall have to dispense with bulky remedies and rely upon remedies which are effective in quanti. ties not exceeding a few cubic centimetres.

Mr. Ballance decided that the best way to introduce so large a quantity as, say, 40 c.c. of serum into the cranial subarachnoid space would be via the anterior horn of the lateral ventricle, by means of a cannula passed along the side of the falx cerebri through the corpus callosum; and this operation he performed successfully on a case of general paralysis on Feb. 25th. The patient made a rapid recovery. The operation was repeated on March IIth. and this time five drops of Fowler's solution were added to the serum. Convalescence on this second occasion was attended by slight mental disturbance, the result, I am inclined to think, of the Fowler's solution. There is no doubt that this patient's. mental state has improved since these operations, but how far the improvement is the result of the treatment it is yet too early to say. Mr. Ballance has twice successfully performed the same operation on a second case of general paralysis. In this case the cortical degeneration had already reached an advanced stage, and I am not satisfied that any substantial improvement in the mental condition has been effected.

The intraventricular operation was undertaken in the belief that by its means a considerable pro. portion of the serum introduced is enabled to reach the upper cranial chamber, where it can circulate freely over the cerebral cortex. We have seen, however, that most, if not all, of the intraventricular cerebro-spinal fluid escapes through the openings of the fourth ventricle into the subarachnoid space of the inferior cranial chamber, and that thereafter it tends to pass downwards through the foramen magnum, rather than upwards through the incisura tentorii. This suggests that the trend of the serum injected intraventricularly is in the same direction. For this reason it is advisable to keep the head low after the intraventricular operation so as to favour the passage of the fluid through the incisura; but even assuming that only a small proportion of the serum injected intraventricularly reaches the cerebral cortex, we may, I think, safely conclude that much more reaches it by this method than by the intraspinal one, owing to the much greater supply of serum which reaches the inferior cranial chamber by 
the intraventricular method. The most satisfactory way of ascertaining to what extent the intraventricular cerebro-spinal fluid reaches the cerebral cortex would be by injecting the ventricles with colloidal carbon, an experiment which Dr. Golla has not been able to carry out with the animals at his disposal.

I have said that in tabes spinal injections usually suffice, for by their means the whole of the affected area of the central nervous system can be reached by the injections. There is, however, one form of tabes in which the question of resorting to intracranial injections presents itself-that form, namely, in which optic atrophy, generally of a rapidly progressive character, occurs. There is every reason to believe that the atrophy in these cases is due to the presence of spirochætes in the immediate neighbourhood of the optic nerres. It should be observed that the arachnoid membrane forms but a loose investment round the optic nerves, and thus allows the cerebro-spinal fluid to circulate freely about them. Dr. Golla's experiments have shown that when colloidal carbon is introduced subdurally into the upper cranial chamber the walls of the peri-optic subarachnoid space are blackened with carbon particles, and, moreover, that these can actually be traced into the nerves themselves. Now, it is true that the optic nerves can be reached by intraspinal injections, but certainly not so effectively as by the intracranial route. It was for this reason that I sought the help of Mr. Ballance in a case of tabetic optic atrophy with com. plete blindness of one eye and almost complete blindness of the other. This was the case in which the attempt was made to introduce the serum subdurally, and in which it was found impossible to get more than a very small quantity to remain under the membranes. Directly the cannula was removed, almost the whole of what had been introduced escaped through the opening in the dura. In a subsequent operation the intraventricular method was adopted, and there can, I think, be little doubt that this method affords the most effective way of reaching the optic nerves, for the serum rapidly passes into the peri-optic sub. arachnoid spaces, where it can soak into the optic nerves. The patient recovered from the operations, but the optic neurons had already been injured beyond recovery, and he has since lost the small degree of sight that was left to him at the time of the second operation. Nevertheless, it seems reasonable to hope that we may be able to arrest these cases of optic atrophy by means of suitable intra. cranial injections.

A word of caution in conclusion. The plan of treating syphilitic diseases of the central nervous system by the introduction of remedies into the cerebro-spinal fluid opens a new chapter in therapeutics, and has, I believe, a great future before it; but it is one beset with many dangers, and needs to be carried out with the utmost caution, not only in regard to strict asepsis - that goes without sayingbut also as regards the remedies employed, and the strength, number, and intervals of the injections. It must not be forgotten that in introducing remedies into the cerebro-spinal fluid we are, as it were, going behind one of nature's defences-the capillaries of the central nervous system. When, e.g., arsenic is administered intravenously, intramuscularly, or by the mouth, it does not reach the neurons of the central nerrous system; it is prerented from reaching them by the local capillaries, which constitute an important line of defence against poison circulating in the blood. When, therefore, it is introduced into the cerebro-spinal fluid only very minute doses shall be employed. Wimpole-street, $W$.

\section{THE INTRATHECAL INJECTION OF SALVARSANISED SERUM.}

By GoRdon W. SPENCER, B.A., M.B., B.C. Cantab., HOUSE PHYSICIAN, BRISTOL ROYAL TKEIRMARY.

THE diseases of tabes dorsalis and general paralysis of the insane were, until quite recently, always looked upon as being "parasyphilitic" in origin, or, in other words, diseases which follow on in the wake of syphilis rather than being definitely attributable to it. Following the discovery of the spirochreta pallida by Schaudinn and the successful inoculation of anthropoid apes by Metchnikoff, Neisser, and others, the researches of Mott, Noguchi, ${ }^{2}$ Marie, ${ }^{3}$ and Levaditi, who have found the spirochæta pallida in films from the brains of general paralytics, have conclusively proved the syphilitic origin of the diseases in question.

These diseases were usually treated with mercury and potassium iodide; very little benefit was derived from this or other antisyphilitic treatment, but the reason for the disease not responding to treatment was not at first obvious. An explanation has been found in the selective action of the choroid plexus, which allows practically no drugs. or antibodies to pass through into the cerebrospinal fluid. In proof of this are the facts: (a) that the spinal cord and fluid do not become stained in jaundice; (b) that mercury and arsenic are not present, or only in minute traces after the patient has been taking the drug for some time; and (c) that if trypan blue be injected intravenously the cord or fluid does not become stained. It is, therefore, obvious that if we are to reach the spirochætes, which we now know are present in the brain and cord, we must place our antisyphilitic remedies into the cerebro-spinal fluid itself.

Intravenous injections of salvarsan and neosalvarsan having failed to give relief it occurred to Dr. Swift, ${ }^{4}$ of the Rockefeller Institute, to try the intrathecal injection of the serum of a patient who had had salvarsan. Nierenstein, $\check{5}$ working with atoxyl, has shown that arsenic by itself has no effect upon trypanosomes in vitro. Upon adding protein death occurred after a certain time, but this time was much lessened if the atoxyl was allowed to combine with the protein first and then added to the trypanosomes. This then provides the rationale of our present treatment, as there is only a minute quantity of protein in the cerebrospinal fluid; so the neosalvarsan is given intravenously first, and then the serum derived from the patient, which will therefore contain the arsenicprotein compound, is injected intrathecally.

Technique.-An intravenous injection of neosalvarsan 0.75 grm. is first given. In this series of experiments the neosalvarsan has been given in concentrated solution, never more than 10 c.c. of doubly distilled water being used to dissolve it, and the injection has been made with a "record" syringe without previously dissecting out the vein. The two main points to notice are: (1) the im. portance of using doubly distilled water, which almost reduces to nothing the reaction to the drug; and (2) that the injection should be made by 